\title{
BMJ Open Sickness absence, disability pension and economic situation after a spontaneous subarachnoid haemorrhage among people of working age: a Swedish longitudinal nationwide cohort study
}

\author{
Elisabeth Ronne-Engström (1) , ${ }^{1}$ Kristina Alexanderson, ${ }^{2}$ Emilie Friberg ${ }^{2}$
}

To cite: Ronne-Engström E, Alexanderson K, Friberg E. Sickness absence, disability pension and economic situation after a spontaneous subarachnoid haemorrhage among people of working age: a Swedish longitudinal nationwide cohort study. BMJ Open 2021;11:e040941. doi:10.1136/ bmjopen-2020-040941

- Prepublication history for this paper is available online To view these files, please visit the journal online (http://dx.doi org/10.1136/bmjopen-2020040941).

Received 26 May 2020 Revised 13 November 2020 Accepted 23 November 2020

Check for updates

(C) Author(s) (or their employer(s)) 2021. Re-use permitted under CC BY-NC. No commercial re-use. See rights and permissions. Published by BMJ.

${ }^{1}$ Department of Neuroscience, Neurosurgery, Uppsala University, SE-751 85 Uppsala, Sweden

${ }^{2}$ Department of Clinical Neuroscience, Division of Insurance Medicine, Karolinska Institute, SE-171 77 Stockholm, Sweden

Correspondence to Dr Elisabeth Ronne-Engström; elisabeth.ronne-engstrom@ neuro.uu.se

\section{ABSTRACT}

Objectives The aim was to describe the course of sickness absence (SA), disability pension (DP) and workrelated economic situation defined as earnings (EA) and disposable income (DI), after spontaneous subarachnoid haemorrhage (SAH). Associations of SA, DP, EA and DI with demographic factors were also studied.

Design A longitudinal cohort study of all 1932 people in Sweden who in January 2005 to December 2010 had a first time SAH when aged 17 to 64 years and survived during the 3-year follow-up. Microdata from four nationwide administrative registers were used.

Main outcome measures Primary outcome was the presence of SA and DP and how this changed during the study period of 5 years (the year before, the year of SAH and the following 3 years). The secondary outcome was the development of the income variables EA and DI. Demographic factors analysed were sex, age, source of bleeding, country of birth, family situation, educational level and type of living area.

Results The year before the SAH, $7.9 \%$ of women and $4.6 \%$ of men had some $S A$ registered $(p<0.004)$. A model consisting of female sex, higher education and living single predicted having SA that year. At the end of the follow-up, $39.2 \%$ of women and $28.3 \%$ of men had SA and/or DP $(p<0.0001)$. A model consisting of female sex, living in a village/ rural area and having a defined bleeding source for the SAH was predicting having SA and/or DP at end of follow-up. The levels of EA decreased, while DI increased during follow-up and were at the end of followup associated with age, sex, type of living area, country of birth, educational level and family situation. The women's EA was lower than the men's during all years.

Conclusions SAH influenced future SA, DP, as well as EA. Both SA, DP and the economic variables studied were predicted by models including sex.

\section{INTRODUCTION}

Spontaneous subarachnoid haemorrhage $(\mathrm{SAH})$ is a condition with high morbidity and mortality (see for example, ${ }^{1}$ ). SAH is
Strengths and limitations of this study

- The data used is from nationwide registers of high quality and with no dropouts.

- The information about sickness absence and disability pension was provided in exact dates.

- The economic information was provided for calendar years.

- The study does not include information about the possible effects of the neurological condition after subarachnoid haemorrhage.

responsible for 5 per cent of stroke incidents and affects a younger population than ischaemic stroke. The mean age at the start of the disease is 55 to 60 years (see for example ${ }^{2}$ ). In two-third of the cases, the cause of the SAH is an aneurysm on the cerebral blood vessels. Most treatment guidelines are focussed on early detection and elimination of the bleeding source, in order to avoid repeated bleedings which can be deadly. During the first few weeks after the SAH, there are substantial changes in the brain's blood flow and metabolism, with vasospasm, being a major problem. The patients are during this time usually treated at neuro-intensive or neuro-intermediate care units. After discharge from neurosurgical units, patients are usually treated within internal medicine or neurology before returning home. The need for, as well as the availability of, rehabilitation varies considerably. The main literature on SAH is mostly concerned with the pathophysiology and the acute management. The outcome after SAH has mostly been measured in mortality or regarding functional outcomes, measured with, for example, modified Rankin Scale. ${ }^{3}$ More recently, 
however, studies have been published about residual symptoms and health-related quality of life. Patients with SAH often suffer from various combinations of neurological deficits and cognitive symptoms that create difficulties in returning to previous life situation. ${ }^{4-8}$ Return to work has been studied regarding the influence of cognitive effects, ${ }^{910}$ and is associated with higher life satisfaction (see for example, ${ }^{11}$ ). It is reasonable to assume that SAH also affects work capacity, which would be reflected in increased sickness absence (SA) and disability pension (DP), and a worsened economic situation. In order to improve the outcome of vocational rehabilitation, it could be important to consider the socioeconomic situation after SAH. The most important factors for vocational rehabilitation are probably age and neurological outcome, but sex, educational level and family situation preceding the SAH could also contribute. ${ }^{12}$ However, the scientific knowledge about this is limited. To our knowledge, the present paper is the first report on SA, DP and economic conditions after SAH.

The aim was to describe SA, DP and the work-related economic situation defined as earnings (EA), that is, sum of salary and other work-related economic benefits, and disposable income (DI), that is, the net money that the individual can use for spending and saving, over several years, among people with an incident SAH. Further, to explore the influence of certain demographic factors on SA, DP, EA and DI.

\section{METHODS}

A longitudinal cohort study was conducted, based on anonymised microdata from four nationwide administrative registers linked at the individual level by use of the individual personal identity number assigned all people registered as living in Sweden. ${ }^{13}$ Information from the following registers was used: the Board of Health and Welfare: the inpatient register with information on all hospitalisations (dates and diagnoses) and the cause of death register (dates); Statistics Sweden: the longitudinal database for income and labour market studies (LISA) (information on demographic factors from the calendar year before the SAH and annual information on types and amounts of incomes and benefits, including old-age pension (OAP)), and from the Swedish Social Insurance Agency's microdata for the analysis of social insurance register (MiDAS) information about dates and duration of SA and DP.

Initial inclusion criteria were: being admitted to a hospital with a first time spontaneous SAH (diagnosis I60.0-I60.9 according to the International Statistical Classification of Diseases and Related Health Problems version 10, ICD-10) ${ }^{14}$ sometime during the 6years January 2005 to December 2010 when aged 17 to 64 years $(n=3243)$. In order to study the effect of the SAH we excluded 736 individuals already being on DP during the year before the SAH date. Furthermore, 563 of those not on DP the year before the SAH date, died within the follow-up period and were excluded, the majority of them (87\%) died within 6 weeks. Finally, 12 individuals emigrated and could not be followed, leaving 1932 people for the study.

Five years were studied; Year -1 was the year before the SAH, Year 0 during which the SAH occurred, and the three following years (Years 1 to 3) was the follow-up period. When calculating SA and DP, Year 0 started with the exact date of the SAH. In the calculations concerning incomes, calendar years were used and SAH then occurred sometime during Year 0. Primary outcome was having had any SA or DP time during each of the five studied years. Furthermore, the income variables EA and DI were studied. EA was defined as the sum of the income from work and other economic compensations payed by the employer. DI includes in addition to EA, also other types of benefits and incomes, for example, SA, DP, welfare and housing benefits, and is the net amount of money that can be used by the individual for consumption. It was also noted if economic compensation for OAP (a monthly compensation) was paid in Year -1 and Year 3. For the economic variables we compared the changes over time.

The following demographic variables were included: sex (women vs men), source of SAH (defined artery (ICD-10 I60.0- I60.8) vs unknown (I60.9)), birth country (Nordic countries (Sweden, Norway, Denmark, Finland or Iceland) vs the rest of the world), family situation (single vs married/cohabitant) and educational level (high school/university vs elementary school). The type of living area during the year before the SAH was defined by the density of the population as big or medium-sized/ small cities versus villages/rural. Age at the year of SAH was used.

For the graphical presentation of the change of economic variables, the mean \pm 0.95 CI was used. For graphical comparison of the DI with the general population we used official population statistics for women and men aged 16 to 64, available at Statistics Sweden's website. ${ }^{15}$ The time-period 2007 to 2011 was chosen since this was in the middle of our study period. The general population data were adjusted according to the median age and sex distribution in our cohort. No statistical comparison was conducted since only median values were available.

\section{Social security in Sweden}

All people in Sweden aged 16 and above with income from work or unemployment benefits are covered by the public SA scheme. The employer pays the first 2 weeks of a SA spell. Thereafter, the Social Insurance Agency pays their SA benefits from day 15 onwards and for unemployed from day 2. DP can be granted to all people aged 19 to 64 with long-term or permanent work incapacity due to morbidity and is also paid by the Social Insurance Agency. DP and SA can be granted in different levels: $25 \%, 50 \%$, $75 \%$ and $100 \%$ of ordinary work hours. The SA compensation is about $80 \%$ and DP compensation $65 \%$ of the income loss, up to a certain level. The rules for SA were changed in July 2008 with an introduction of a maximum 
time for SA spells of 2.5 years. We did not control for this. Customary old-age pension age is 65 years, but it is possible to retire earlier, as well as to retire part-time.

D

\section{Patient and public involvement}

This research was a register study, done without patient or public involvement. Data were provided anonymised.

\section{Statistical analyses}

Independent t-test was used for the univariate comparisons and paired t-test when comparing income variables in the beginning and the end of the study period. Spearman rank was used for correlations and $\chi 2$ for comparing distributions. Multivariate analysis was done with general linear/non-linear modelling of best subsets of variables according to Akaike's criterion. ${ }^{16}$ All the variables, that is, sex, level of education, country of birth, type of living area, family situation, age and bleeding source were included in all the multivariate analyses. The motive for including the bleeding source in the analyse of EA and DI Year -1 was that a defined bleeding source (usually an aneurysm) is frequently seen together with other health issues, for example, hypertension, smoking and alcohol use. A p value $<0.05$ was considered significant. Statistica 13, Dell Inc, (Tulsa, Oklahoma) was used for the statistical analyses.

\section{RESULTS}

In the cohort of 1932 individuals with incident SAH when aged 17 to 64 years, the median age was 51 years (IQR 43 to 58$), 56.2 \%$ were women and $57.9 \%$ had a defined source of bleeding. Furthermore, $88.5 \%$ were born in Nordic countries, $40.5 \%$ were single, $78.6 \%$ had an educational level of high school or university and $69.0 \%$ lived in big or medium-sized cities (table 1). The highest number of individuals with SA was seen in Year 0, which started with the day of SAH. However, $20.2 \%$ did not have any SA or DP that year (figure 1). The number of people with any SA decreased during the follow-up period while the numbers with any DP, as well as with combinations of SA and DP increased (figure 1). During Year -1 , the year before the SAH date, $7.9 \%$ of women and $4.6 \%$ of men had some SA registered $(\mathrm{p}<0.004)$. The multivariate analyses showed that a model with the combination of female sex, having higher education and living single best predicted having SA the year before the date of SAH $(\mathrm{p}<0.0005)$. Year 3 , that is, at the end of the follow-up, $39.2 \%$ of women and $28.3 \%$ of men had SA and/or DP $(p<0.0001)$. The multivariate analyses for Year 3 showed that the combination of female sex, living in small city or village and having a defined bleeding source best predicted having SA and/or DP $(\mathrm{p}<0.0001)$.

The incomes measures, EA and DI during each calendar Year -1 to Year 3, stratified by sex, are shown in figure 2. The income variables displayed similar patterns for women and men but were significantly
Table 1 Demographic information about the studied cohort of 1932 individuals

\begin{tabular}{ll}
\hline Age years (median, IQR) & 51 (43 to 58) \\
\hline Sex & $56.2 \%$ \\
\hline Women & $43.8 \%$ \\
\hline Men & \\
\hline Bleeding source & $57.9 \%$ \\
\hline Defined (ICD-10 I60.1-I60.8) & $42.1 \%$ \\
\hline Undefined (I60.9) & \\
\hline Country of birth & $88.5 \%$ \\
\hline Nordic countries & $11.5 \%$ \\
\hline Rest of the world & \\
\hline Family situation & $40.5 \%$ \\
\hline Single & $59.5 \%$ \\
\hline Married/cohabitant & \\
\hline Education & $78.6 \%$ \\
\hline High school/university & $21.4 \%$ \\
\hline Elementary & $69.0 \%$ \\
\hline Type of living area & $31.0 \%$ \\
\hline Big/medium size cities & \\
\hline Small cities, villages, rural & \\
\hline
\end{tabular}

ICD-10, International Statistical Classification of Diseases and Related Health Problems, version 10.

lower for women (see also table 2). The EA showed a continuous decrease during the follow-up period, while DI gradually increased. The decrease in EA and increase in DI were significant both for women and men (EA $p<0.00001$ for both sexes and DI $p<0.002$ for women and $\mathrm{p}<0.05$ for men). The DI in the patient cohort and

Distribution of Sickness Absence (SA) and Disability Pension (DP) after Spontaneous Subarachnoid Haemorrhage $(n=1932)$

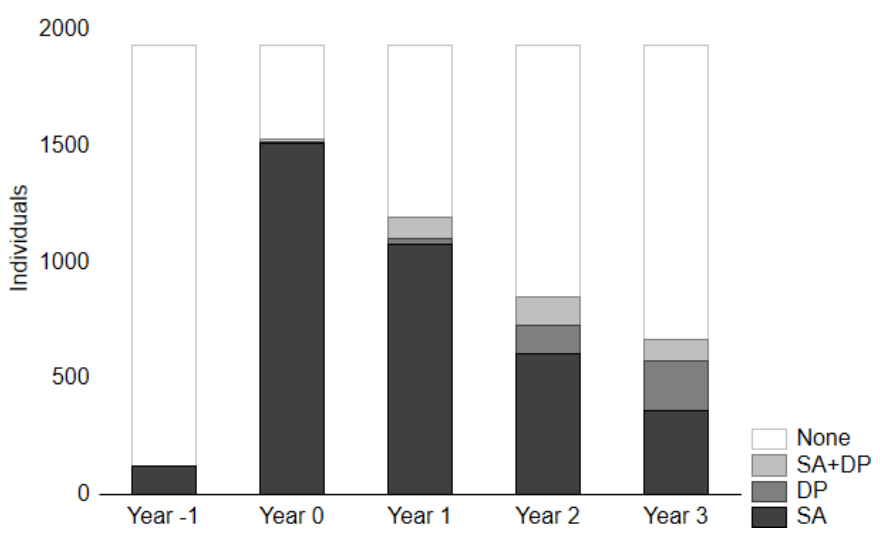

Figure 1 The figure shows the number of individuals with at least some SA, DP, alone or in combination (SA+DP), during the study period, among individuals of working age with a first subarachnoid haemorrhage (SAH) and not on DP in the year before SAH date. Year 0 started with the date of the $\mathrm{SAH}$. 'None' indicates that no SA or DP was registered. 


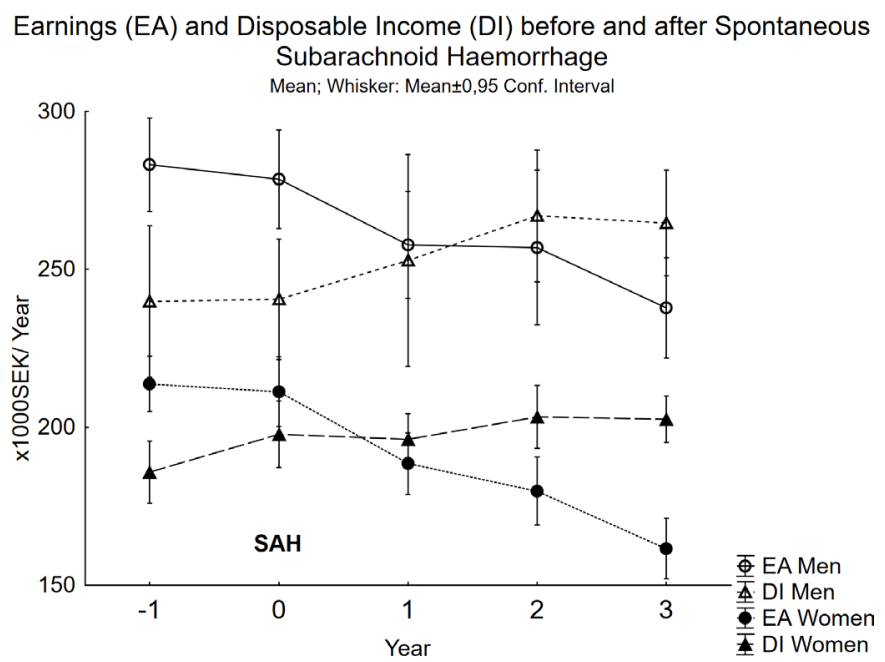

Figure 2 The y-axis shows the income/year from EA and $\mathrm{DI}$ in the cohort of individuals of working age with a first subarachnoid haemorrhage (SAH). Income was displayed as 1000 Swedish crowns (SEK)/year. Year 0 is the calendar year during which the SAH occurred. In the figure, the graphs were stratified by sex. Conf.,confidence.

in the general population is displayed in figure 3 . The two groups followed a similar pattern, with a gradual increase over the years, but with a steeper slope for the general population.

The impact of the demographic variables on EA and DI at the beginning and at the end of the study period (Year -1 and Year 3) was studied first with univariate analyses (table 2). Women, those with a lower educational level, and individuals born outside Nordic countries had significantly lower income levels compared with men, those with higher educational level and those born in Nordic countries, respectively. Individuals with a defined bleeding source had lower EA and DI at Year 3 compared with those with unknown source. Those who were single had lower EA in Year -1 and Year 3 and DI in Year 3. The same pattern was seen for those living in small cities/rural areas compared with in big/ medium-sized cities. Finally, there was a weak but significant positive correlation between age and EA Year -1 , DI Year -1 and DI Year $3(0.11,0.17$ and 0.05 , respectively), and a significant negative correlation $(-0.15)$ between age and EA Year 3 .

For the multivariate analyses, the variables used in the univariate analyses were entered in the model, together with age at SAH. Table 3 shows the best subsets of variables predicting the EA and DI in Year -1 and Year 3. Sex, educational level, country of birth and age were included in all the four models. For EA and DI in Year 3 also SAH source was included in the best predicting model. Type of living area and family situation were included in the best predicting models for EA Year -1 and Year 3 and DI Year 3.

Economic compensation for OAP was registered the calendar year before the SAH for $8.1 \%$ of women and $5.5 \%$ of men $(\mathrm{p}<0.03)$ and Year 3 for $23.7 \%$ of women
Table 2 Univariate analysis comparing the income measures earnings (EA) and disposable income (DI) by the dichotomised demographic variables

\begin{tabular}{|c|c|c|c|c|}
\hline \multicolumn{4}{|c|}{ Year } & \multirow[t]{2}{*}{$P$ value } \\
\hline \multicolumn{4}{|c|}{ Sex } & \\
\hline & & Women & Men & \\
\hline \multirow[t]{2}{*}{ EA } & -1 & $214 \pm 147$ & $283 \pm 220$ & $<0.0001$ \\
\hline & 3 & $162 \pm 161$ & $238 \pm 236$ & $<0.0001$ \\
\hline \multirow[t]{2}{*}{ DI } & -1 & $186 \pm 164$ & $240 \pm 355$ & $<0.0001$ \\
\hline & 3 & $203 \pm 123$ & $265 \pm 248$ & $<0.0001$ \\
\hline \multicolumn{5}{|c|}{ Bleeding source } \\
\hline & & Aneurysm & Unknown & \\
\hline \multirow[t]{2}{*}{ EA } & -1 & $242 \pm 171$ & $247 \pm 204$ & NS \\
\hline & 3 & $178 \pm 200$ & $219 \pm 200$ & $<0.0001$ \\
\hline \multirow[t]{2}{*}{ DI } & -1 & $204 \pm 175$ & $217 \pm 356$ & NS \\
\hline & 3 & $222 \pm 185$ & $241 \pm 199$ & $<0.03$ \\
\hline \multicolumn{5}{|c|}{ Country of birth } \\
\hline & & $\begin{array}{l}\text { Nordic } \\
\text { countries }\end{array}$ & $\begin{array}{l}\text { Rest of the } \\
\text { world }\end{array}$ & \\
\hline \multirow[t]{2}{*}{ EA } & -1 & $253 \pm 187$ & $158 \pm 154$ & $<0.0001$ \\
\hline & 3 & $203 \pm 204$ & $131 \pm 162$ & $<0.0001$ \\
\hline \multirow[t]{2}{*}{ DI } & -1 & $218 \pm 278$ & $142 \pm 143$ & $<0.0001$ \\
\hline & 3 & $238 \pm 198$ & $165 \pm 105$ & $<0.0001$ \\
\hline \multicolumn{5}{|c|}{ Family situation } \\
\hline & & Single & $\begin{array}{l}\text { Married/ } \\
\text { cohabitant }\end{array}$ & \\
\hline \multirow[t]{2}{*}{ EA } & -1 & $220 \pm 187$ & $261 \pm 183$ & $<0.0001$ \\
\hline & 3 & $178 \pm 181$ & $206 \pm 212$ & $<0.004$ \\
\hline \multirow[t]{2}{*}{ DI } & -1 & $208 \pm 360$ & $211 \pm 177$ & NS \\
\hline & 3 & $217 \pm 173$ & $239 \pm 202$ & $<0.02$ \\
\hline
\end{tabular}

\begin{tabular}{|c|c|c|c|c|}
\hline \multicolumn{5}{|c|}{ Education } \\
\hline & & $\begin{array}{l}\text { University/ } \\
\text { high school }\end{array}$ & $\begin{array}{l}\text { Elementary } \\
\text { school }\end{array}$ & \\
\hline \multirow[t]{2}{*}{ EA } & -1 & $259 \pm 189$ & $190 \pm 162$ & $<0.0001$ \\
\hline & 3 & $212 \pm 207$ & $131 \pm 161$ & $<0.0001$ \\
\hline \multirow[t]{2}{*}{ DI } & -1 & $218 \pm 287$ & $177 \pm 166$ & $<0.006$ \\
\hline & 3 & $242 \pm 204$ & $185 \pm 125$ & $<0.0001$ \\
\hline \multicolumn{5}{|c|}{ Type of living area } \\
\hline & & $\begin{array}{l}\text { Big/medium } \\
\text { cities }\end{array}$ & $\begin{array}{l}\text { Small cities/ } \\
\text { village/rural }\end{array}$ & \\
\hline \multirow[t]{2}{*}{ EA } & -1 & $252 \pm 199$ & $226 \pm 152$ & $<0.005$ \\
\hline & 3 & $208 \pm 212$ & $167 \pm 171$ & $<0.0001$ \\
\hline \multirow[t]{2}{*}{ DI } & -1 & $208 \pm 202$ & $212 \pm 372$ & NS \\
\hline & 3 & $237 \pm 196$ & $215 \pm 180$ & $<0.02$ \\
\hline
\end{tabular}

Income was measured x 1000SEK/year, (mean $\pm 1 S D)$. Analysis was done for Year -1 (ie, the calendar year before the SAH) and Year 3, that is, the last calendar year of the follow-up period.

$\mathrm{SAH}$, subarachnoid haemorrhage; SEK, Swedish crowns. 


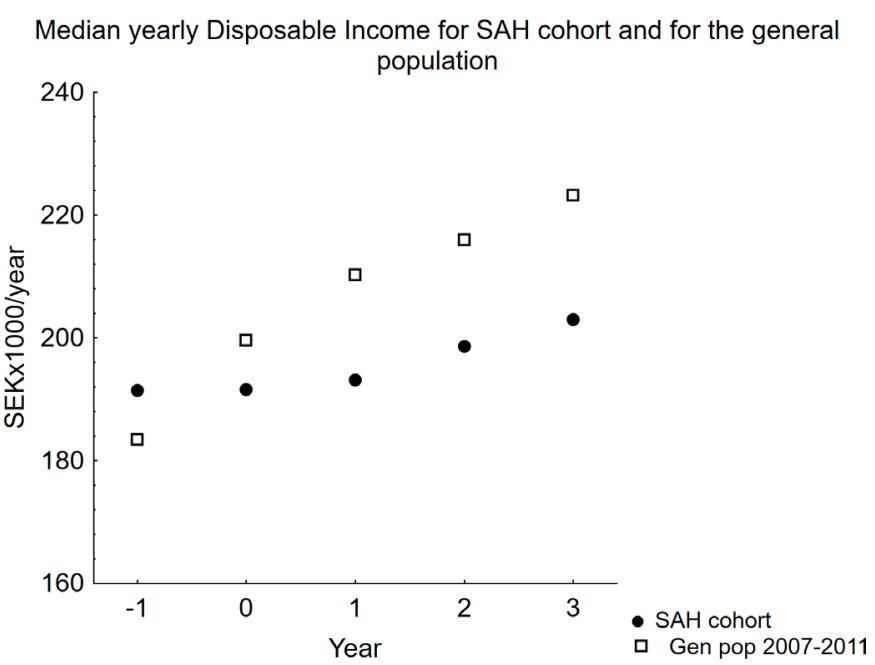

Figure 3 Yearly median disposable income (DI) for the SAH cohort and for the general population of working age. For displaying the DI of the general population, the time period of 2007 to 2011 was chosen since this was in the middle of our study period. Genpop, general population; SAH, subarachnoid haemorrhage;SEK, Swedish crowns.

and $24.3 \%$ of men. Finally, $7.5 \%$ of the cohort had no registered SA, DP, EA or OAP at the end of the follow-up. However, $86 \%$ of them had a positive DI development.

\section{DISCUSSION}

\section{Principal findings}

In this Swedish nationwide longitudinal cohort study of all people of working age with a first known spontaneous SAH and not on DP at the SAH date, and who survived for 3 years, we explored their SA, DP and incomes during 1 year prior to SAH through 3 years after.

Significantly more women than men had at least some SA before and SA/DP at the end of the follow-up. The reasons for this could be differences in how SAH is expressed in women and men. A gender difference in comorbidities has been shown for some other conditions, for example, diabetes. ${ }^{17}$ This needs to be further studied in SAH. There could also be diverse needs for SA due to type of occupation in the very gender segregated labour market ${ }^{18}$ and possible gender-differences in handling SA of patients. ${ }^{19}$

The year following the SAH date was when the highest number of individuals in the cohort had at least some SA, as would be expected. However, $22.7 \%$ had no documented SA or DP in that year. There could be various reasons for this. Some may not have been in paid work and eligible for SA. Others might already be on OAP. It could also include individuals with a favourable clinical course and a short SA covered by the employer (which we do not have information about). During the following years, the number of individuals with at least some SA gradually decreased and number of individuals on combinations of SA and DP increased. This could signal a permanent work incapacity and/or exit from the labour market. The higher rates of people receiving OAP the last calendar year of the follow-up could be a result of increasing age but also be a way to compensate for perceived lack of work capacity, for example, if not granted SA or DP benefits.

In this cohort, EA decreased during follow-up and did not reach the levels in the calendar year before the SAH. This could be a result both of lower work capacity and also of exiting the labour market. DI increased during the study period, as in the general population, but with a less steep slope. DI is the net amount of money available for spending and saving after expenses, for example, after income taxes were paid. Similar patterns have been observed for people with other diagnoses, for example, multiple sclerosis, ${ }^{20}$ and could be related to the Swedish social security system. The results need to be compared with those from similar cohorts in other countries.

Previous studies examining cost of illness related to SAH have primarily focussed on the medical costs (see for example, ${ }^{21}$ ). Rivero-Arias et al included the economic burden of aneurysmal SAH in UK, and non-healthcare costs including informal care and productivity loss. ${ }^{22}$ Here we studied the changes in income for all survivors of spontaneous SAH of working age, since we think that this information could be useful in vocational rehabilitation measures. We excluded those already on DP the year before the SAH since other conditions could interfere with returning to work.

Table 3 Best subset of variables predicting earnings and disposable income, in Year -1 and Year 3

\begin{tabular}{lllll}
\hline & Earnings & & Disposable income & \\
\cline { 2 - 3 } Variables & Year $\mathbf{- 1}$ & Year $\mathbf{3}$ & Year $\mathbf{- 1}$ & Year $\mathbf{3}$ \\
\hline 1 & Sex & Sex & Sex & Sex \\
\hline 3 & Education & Education & Education & Education \\
3 & Country of birth & Country of birth & Country of birth & Country of birth \\
5 & Living area & Living area & Age & Living area \\
6 & Family situation & Family situation & & Family situation \\
7 & Age & Bleeding source & & Bleeding source \\
\hline
\end{tabular}

All four subsets were significant at a $p$ level of $<0.000001$. 
A potential confounding factor in income analyses among individuals with SAH is sex. In Sweden, women had $72 \%$ and $75 \%$ of men's EA in year 2000 and 2015, respectively. ${ }^{15}$ The clinical course of spontaneous SAH is also related to sex, since most of the aneurysmal bleedings with a more serious course occur in women (see for example,$\left.{ }^{23}\right)$. This is in line with our results that showed lower incomes during all years for women. The model best predicting EA at Year - 1 included sex, educational level, country of birth, type of living area, family situation and age. The best model predicting DI Year -1 included sex, educational level, country of birth and age. At Year 3, type of living area, family situation and bleeding source were included in the most predictive model. That is, at Year 3, also bleeding source became a variable in the best model. This was expected since those with undefined bleeding source frequently have a more favourable clinical course and are more likely to return to work. This is consistent with the notion that social factors are also important contributors to rehabilitation and return to work generally, in line with results from previous studies. ${ }^{121524}$

\section{Strength and limitations}

A strength of the study is that it included all incident patients with SAH in Sweden during the study period fulfilling the inclusion criteria, rather than a subset treated at a particular clinic, and that the information used was from nationwide registers with high quality and no dropouts. ${ }^{25}{ }^{26}$ Another strength is that no selfreports, possibly biassed by, for example, recall bias, were used. It is a strength that the information about SA and DP was available with exact dates. However, a limitation is that information about SA only was available for those that received economic compensation from the Social Insurance Agency, that is, among those employed people with SA spells $\leq 14$ days were not included. However, this can also be seen as a strength, as not the short-term SA, for example, due to common cold were included. It is also a limitation that information about incomes was available only for each calendar year, rather than relative to the exact SAH date. However, the main results we report regarding incomes are from the calendar year before and the three calendar years after the year of SAH (ie, the last year of follow-up). One major limitation is that we did not have information about functional neurological outcome which is a crucial factor in vocational rehabilitation. Such information is not yet available at a nationwide level for patients with SAH in Sweden.

\section{CONCLUSION}

We studied SA, DP and the economic situation in a nationwide cohort of patients with spontaneous $\mathrm{SAH}$ at working age. The main results showed that the proportion of people with at least some SA was highest during the year following the SAH date. Initially SA dominated, but was gradually replaced by SA in combination with DP or DP only. Furthermore, EA decreased while DI increased, the latter in parity with the general population. The combination of female sex, living in small city or village and having a defined bleeding source best predicted having SA and/ or DP after SAH.

Contributors ERE formulated the research questions that were based on neurosurgical clinical experiences. ERE further analysed data and wrote the manuscript. EF and KA contributed with their expertise in the area of insurance medicine, epidemiological research and gender medicine. KA contributed with the register data used in the study. Furthermore, EF and KA participated in discussions about the content and did critical revisions of the manuscript.

Funding The study was conducted with research grant support from the Swedish Research Council for Health, Working Life and Welfare (2007-1762) and, the Uppsala University Hospital Clinical Research Funds (ALF) 45201 and the Swedish Research Council (2017-00624). The funding sources had no role in the design and conduct of the study.

Competing interests None declared.

Patient and public involvement Patients and/or the public were not involved in the design, or conduct, or reporting, or dissemination plans of this research.

Patient consent for publication Not required.

Ethics approval The project was approved by the Regional Ethical Review Board in Stockholm, Sweden.

Provenance and peer review Not commissioned; externally peer reviewed.

Data availability statement No data are available. The data used in this study is administered by the Division of Insurance Medicine, Karolinska Institutet, and cannot be made public. According to the General Data Protection Regulation, the Swedish law SFS 2018:218, the Swedish Data Protection Act, the Swedish Ethical Review Act and the Public Access to Information and Secrecy Act, these type of sensitive data can only be made available, after legal review, for researchers who meet the criteria for access to this type of sensitive and confidential data. Readers may contact Professor Kristina Alexanderson (kristina.alexanderson@ki.se) regarding the data.

Open access This is an open access article distributed in accordance with the Creative Commons Attribution Non Commercial (CC BY-NC 4.0) license, which permits others to distribute, remix, adapt, build upon this work non-commercially, and license their derivative works on different terms, provided the original work is properly cited, appropriate credit is given, any changes made indicated, and the use is non-commercial. See: http://creativecommons.org/licenses/by-nc/4.0/.

ORCID iD

Elisabeth Ronne-Engström http://orcid.org/0000-0001-6345-9415

\section{REFERENCES}

1 Sandvei MS, Mathiesen EB, Vatten LJ, et al. Incidence and mortality of aneurysmal subarachnoid hemorrhage in two Norwegian cohorts, 1984-2007. Neurology 2011;77:1833-9.

2 Ronne-Engström E, Borota L, Kothimbakam R, et al. Outcome from spontaneous subarachnoid haemorrhage--results from 20072011 and comparison with our previous series. Ups J Med Sci 2014:119:38-43.

3 van Swieten JC, Koudstaal PJ, Visser MC, et al. Interobserver agreement for the assessment of handicap in stroke patients. Stroke 1988;19:604-7.

4 Mayer SA, Kreiter KT, Copeland D, et al. Global and domain-specific cognitive impairment and outcome after subarachnoid hemorrhage. Neurology 2002;59:1750-8.

5 Schweizer TA, Al-Khindi T, Macdonald RL. Mini-Mental state examination versus Montreal cognitive assessment: rapid assessment tools for cognitive and functional outcome after aneurysmal subarachnoid hemorrhage. $J$ Neurol Sci 2012;316:137-40.

6 Hedlund M, Zetterling M, Ronne-Engström E, et al. Depression and post-traumatic stress disorder after aneurysmal subarachnoid haemorrhage in relation to lifetime psychiatric morbidity. $\mathrm{Br} J$ Neurosurg 2011;25:693-700. 
7 Post MWM, Boosman H, van Zandvoort MM, et al. Development and validation of a short version of the stroke specific quality of life scale. J Neurol Neurosurg Psychiatry 2011;82:283-6.

8 Pritchard C, Foulkes L, Lang DA, et al. Psychosocial outcomes for patients and carers after aneurysmal subarachnoid haemorrhage. $\mathrm{Br}$ J Neurosurg 2001;15:456-63.

9 Wallmark S, Ronne-Engström E, Lundström E. Predicting return to work after subarachnoid hemorrhage using the Montreal cognitive assessment (MoCA). Acta Neurochir 2016;158:233-9.

10 Buunk AM, Spikman JM, Metzemaekers JDM, et al. Return to work after subarachnoid hemorrhage: the influence of cognitive deficits. PLoS One 2019;14:e0220972.

11 Passier PECA, Visser-Meily JMA, Rinkel GJE, et al. Life satisfaction and return to work after aneurysmal subarachnoid hemorrhage. $J$ Stroke Cerebrovasc Dis 2011:20:324-9.

12 Allebeck P, Mastekaasa A. Swedish Council on Technology Assessment in Health Care (SBU). Chapter 5. Risk factors for sick leave - general studies. Scand J Public Health Suppl 2004;63:49-108.

13 Ludvigsson JF, Otterblad-Olausson P, Pettersson BU, et al. The Swedish personal identity number: possibilities and pitfalls in healthcare and medical research. Eur J Epidemiol 2009;24:659-67.

14 World Health Organization. ICD-10 Version:2015, 2015. Available: https://icd.who.int/browse10/2015/en\#/I60-169

15 Statistics Sweden. Statistical database, 2020. Available: http://www. statistikdatabasen.scb.se/pxweb/en/ssd/

16 Akaike $\mathrm{H}$. A new look at the statistical model identification. IEEE Trans Automat Contr 1974;19:716-23.

17 Breyer M-K, Ofenheimer A, Altziebler J, et al. Marked differences in prediabetes- and diabetes-associated comorbidities between men and women-Epidemiological results from a general population-based cohort aged 6-80 years-The LEAD (Lung, hEart, sociAl, boDy) study. Eur J Clin Invest 2020;50:e13207.

18 Gonäs L, Wikman A, Vaez M, et al. Gender segregation of occupations and sustainable employment: a prospective populationbased cohort study. Scand J Public Health 2019;47:348-56.

19 Upmark M, Borg K, Alexanderson K. Gender differences in experiencing negative encounters with healthcare: a study of longterm sickness absentees. Scand J Public Health 2007;35:577-84.

20 Murley C, Mogard O, Wiberg M, et al. Trajectories of disposable income among people of working ages diagnosed with multiple sclerosis: a nationwide register-based cohort study in Sweden 7 years before to 4 years after diagnosis with a population-based reference group. BMJ Open 2018;8:e020392.

21 Dodel R, Winter Y, Ringel F, et al. Cost of illness in subarachnoid hemorrhage: a German longitudinal study. Stroke 2010;41:2918-23.

22 Rivero-Arias O, Gray A, Wolstenholme J. Burden of disease and costs of aneurysmal subarachnoid haemorrhage (aSAH) in the United Kingdom. Cost Eff Resour Alloc 2010;8:6.

23 Ronne-Engström E, Enblad P, Gál G, et al. Patients with spontaneous subarachnoid haemorrhage - presentation of a 10-year hospital series. Br J Neurosurg 2009;23:499-506.

24 Harris C. Factors influencing return to work after aneurysmal subarachnoid hemorrhage. J Neurosci Nurs 2014;46:207-17.

25 Ludvigsson JF, Almqvist C, Bonamy A-KE, et al. Registers of the Swedish total population and their use in medical research. Eur $J$ Epidemiol 2016;31:125-36.

26 Ludvigsson JF, Andersson E, Ekbom A, et al. External review and validation of the Swedish national inpatient register. BMC Public Health 2011;11:450. 